\title{
Lymphadenectomy in pulmonary metastasectomy
}

\author{
Stefan Welter, Varun Gupta, Ioannis Kyritsis \\ Department of Thoracic Surgery, Lung Clinic Hemer, Theo-Funccius-Str. 1, 58675 Hemer, Germany \\ Contributions: (I) Conception and design: S Welter, I Kyritsis; (II) Administrative support: All authors; (III) Provision of study materials or patients: \\ S Welter, I Kyritsis; (IV) Collection and assembly of data: S Welter, I Kyritsis; (V) Data analysis and interpretation: All authors; (VI) Manuscript \\ writing: All authors; (VII) Final approval of manuscript: All authors. \\ Correspondence to: Stefan Welter. Department of Thoracic Surgery, Lungenklinik Hemer, Theo-Funccius-Str. 1, 58675 Hemer, Germany. \\ Email: Stefan.welter@lkhemer.de.
}

\begin{abstract}
Lymph node (LN) removal during pulmonary metastasectomy is a prerequisite to achieve complete resection or at least collect prognostic information, but is not yet generally accepted. On average, the rate of unexpected lymph node involvement (LNI) is less than $10 \%$ in sarcoma, $20 \%$ in colorectal cancer (CRC) and 30\% in renal cell carcinoma (RCC) when radical LN dissection is performed. LNI is a negative prognostic factor and presence of preoperative mediastinal disease usually leads to exclusion of the patient from metastasis surgery. Nonetheless, some authors found excellent prognoses even with mediastinal LNI in colorectal and RCC metastases when radical LN dissection was performed (median survival of 37 and 36 months, respectively). Multiple metastases, central location of the lesion followed by anatomical resections are associated with a higher LNI rate. The real prognostic influence of systematic LN dissection remains unclear. Two positive effects were described after radical lymphadenectomy: a trend for improved survival in RCC patients and a reduction of mediastinal recurrences from $23 \%$ to $0 \%$ in CRC patients. Unfortunately, there is a great number of studies that do not demonstrate any positive effect of lymphadenectomy during pulmonary metastasectomy except a pseudo stage migration effect. Future studies should not only focus on survival, but also on local and LN recurrence.
\end{abstract}

Keywords: Pulmonary metastasectomy; lymph node dissection; lymph node sampling

Submitted Mar 24, 2020. Accepted for publication Apr 16, 2020.

doi: $10.21037 /$ jtd.2020.04.09

View this article at: http://dx.doi.org/10.21037/jtd.2020.04.09

\section{Introduction}

Pulmonary metastasectomy is increasingly used as local treatment for lung only metastases of solid tumors in stage IV disease to increase overall survival (OS) or at least to interrupt further malignant spread from the lungs to other organs. Lymph node (LN) dissection is well established in primary lung cancer surgery and has important impact on the patient's prognosis $(1,2)$. LN removal during pulmonary metastasectomy is not generally accepted by the European Society of Thoracic Surgeons (ESTS) members. A 2008 survey amongst ESTS members revealed that only $32.2 \%$ surgeons perform LN dissection/excision during metastasectomy and systematic dissection is performed only from $13 \%$ of all responders (3). There is a widespread debate and discussion regarding various strategies ranging from radical $\mathrm{LN}$ dissection in every case to sampling of enlarged LNs to no removal at all (2). Arguments for not performing any LN removal are in most part related to the stage of the disease, which is stage IV; and a further upstaging beyond stage IV after detection of LNI to something like a "stage V" is not possible. Furthermore, the next step in the disease progress is the dissemination of tumor cells from the lung metastasis (LM) into regional LNs and it cannot be controlled or hindered by surgical means. Moreover, there is additional morbidity related with LN dissection (4) and may provide no survival benefit. Henceforth, the further course of the LN disease and prognosis is left in the hands of the patient's destiny. On the contrary, there is the hypothesis, that lung metastases as a 

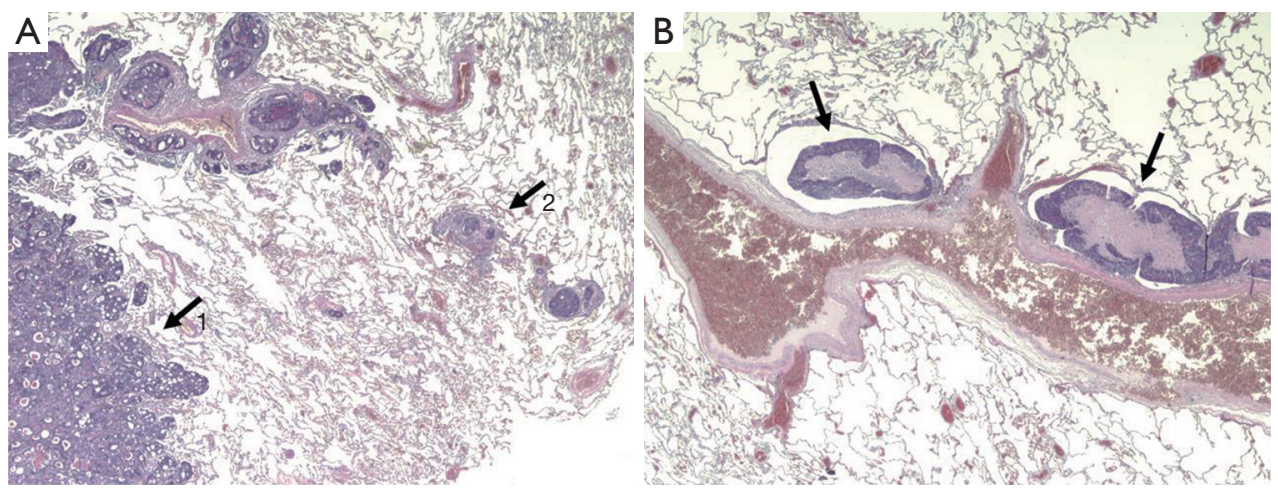

Figure $1 \mathrm{H} \& \mathrm{E}$ stained histologic sections of a rectum cancer lung metastasis demonstrating lymph vessel involvement. (A) $4 \times$ magnification of the main metastasis (arrow 1) and a chain of tumor tissue in lymphatic vessels surrounding a small pulmonary artery branch (arrow 2). (B) $10 \times$ magnification demonstrating tumor tissue inside small lymph vessels (arrows) along a larger pulmonary artery branch.

sole tumor manifestation occurring, with some amount of disease-free survival after removal of the primary tumor, is not a stage IV but rather more similar to a "(multiple) stage I" of the primary tumor. And understandably, in this case a cure is achievable by performing radical resection of these metastases. In contrast to the belief 30 years ago, that lung metastases are incapable of dissemination and every new metastasis originates from the remaining cells of the primary tumor, today it is well accepted that these lung metastases are capable of local, lymphatic as well as hematogenous invasion, proliferation and dissemination $(5,6)$.

\section{Anatomy of the pulmonary lymphatic system}

Most wedge resections as well as enucleations fail to successfully address the lymphatics surrounding the LM and these are thereby left fully or partially intact, serving as a site for possible recurrence. A lobectomy or a pneumonectomy would be an easier alternative ensuring the successful removal of the related lymphatics, but disadvantageously a large amount of healthy lung tissue has to be sacrificed. To confront this problem and decide better resection fields, a thorough understanding of the pulmonary lymphatic system is fundamental.

The lungs contain a large number of lymphatic capillaries located between the alveolar walls and the interlobular, pleural, peribronchial and perivascular connective sheets (Figure 1) (7). This lymphatic system is regarded as a visceral pleural network or parenchymatous peribronchovascular plexus (8). These lymphatic capillaries drain into lymph vessels, "collectors", which contain smooth muscles and one-way valves (9). The segments between two valves are called lymphangions and function as a small pump, facilitating forward movement of the fluid, while preventing retrograde flow. The visceral pleura lymphatic collectors travel along the surface of the lung and anastomose with the lymphatic collectors of the peribronchovascular plexus (bronchial lymphatics) at the lung hilum (8). Along their way, many lymphatic collectors flow into LNs located variably within the lung and the mediastinum. Many of them make the pulmonary, hilar and mediastinal LN chains. Each LN chain acts as a functional unit and may anastomose with the neighboring ones (10). At the end, bronchial lymphatics drain the pulmonary lymph into the venous blood circulation directly by anastomosing into the internal jugulo-subclavian venous confluence (11), or indirectly after connecting with the thoracic duct (12).

One may interpret that malignant nodules in the lungs are always surrounded by lymph vessels and tumor cell distribution may happen in every direction around the lesion, but with a preference to existing lymphatic flow directions along bronchovascular structures to regional LNs and randomly to mediastinal or distant LNs and finally into the venous blood system. Consequently, the following considerations need to be made: (I) safety margins need to be adapted in accordance to the risk for lymph vessel involvement (LVI); (II) Wedge resection or enucleation is inadequate to remove lymphatic structures even in combination with radical lymphadenectomy (LAD); (III) LNI indicate LVI as well and consequently requires anatomical lung resection to remove the lymphatic link between lesion and involved LN.

Extrapulmonary lymphatic connections exist from 
tracheal bifurcation to the pulmonary ligament and some traverse the diaphragm (13) or the hiatal orifice and connect with the aortic LNs of the celiac region or continue into the origin of the thoracic duct (14). Transdiaphragmatic lymphatic connections are also present through the pulmonary ligament. These and the thoracic duct itself may allow tumor cell spread from renal cell cancer (RCC) or colorectal cancer (CRC) liver metastases into mediastinal LNs without involvement of lung tissue (15-17).

\section{Frequency of lymphatic involvement during metastasectomy}

Publications report that LNI rate ranges from 5\% to $32 \%$ $(5,18,19)$. Due to differences in reporting and treatment, a comparison between various publications is difficult. In order to better assess and reach prognostic conclusions, the LNI has to be homogenously reported, and the technique used for $\mathrm{LN}$ evaluation must be reported in extent. The discrepancy in the preoperative $\mathrm{LN}$ evaluation arise as some authors rely only on CT scan (20) whereas others included PET scan, EBUS or mediastinoscopy into preoperative work-up to exclude N2-N3 disease, thereby decreasing the intraoperative "unexpected" findings (21). LN sampling or systematic dissection techniques can vary between authors or even between surgeons in the same team contributing to a publication (22). The number of evaluated nodes are directly associated with the odds ratio of positive nodes in non-small cell lung cancer until 16 extrapulmonary examined nodes (1) and we may expect the same correlation for lung metastases. That implies that the true incidence of LNI can only be detected after radical dissection. This may explain the difference of LNI rate between the international registry of lung metastases (5\%) (18) where a LN sampling or systematic dissection was not performed, in contrast to the higher rates by the second group (32\%) (19) where all patients received a radical LAD. Unexpected LNI rate was $17 \%$ in a newer publication including LAD or LN sampling (23). Other authors did not exclude patients with known mediastinal LNI from pulmonary metastasectomy and thus reported expected and unexpected involvement rates of $44-46.6 \%(24,25)$.

Also, noteworthy is the different local infiltration and spread of pulmonary metastases between different histological cancer subtypes as well as between LNI and LVI (L1). When radical LN dissection is performed, on average, sarcomas report the lowest rates $(10 \%)$ whereas epithelial tumors like breast cancer, CRC (20\%) and RCC
(30\%) illustrate the highest rates $(6,23,26-29)$.

\section{Prognostic impact of lymph node involvement}

Involvement of intrapulmonary, hilar and mediastinal LNs may either represent distant metastases, a lymphatic retroperitoneal or mediastinal spread from the primary or they originate out from the lung metastases (17). A large series including 245 patients with pulmonary metastasectomy and radical LAD demonstrated impaired survival for patients with $\mathrm{N} 1 \mathrm{LNI}$ as compared to no LNI as well as a worse survival for $\mathrm{N} 1+2 \mathrm{LNI}$ in relation to the N0 or N1 group (19). This implies a sequential spread from the pulmonary lesion to $\mathrm{N} 1$ and later $\mathrm{N} 2$ positions with worsening prognosis. For the whole group after R0 metastasectomy the median survival was $63.9,32.7$ and 20.4 months with N0, N1 and N1+2 LNI respectively (19). Significant prognostic deterioration for $\mathrm{N}+$ patients as compared to N0 patients has been reported by many authors $(23,24,26,29,30,31)$. In a French collective, LNI appeared as a significant prognostic factor in both the univariate and multivariate analyses with a survival of 94 months in $\mathrm{N} 0$ and 42 months in $\mathrm{N}+$ patients $(\mathrm{P}<0.0001$; OR $=0.573$ ) (24). A retrospective Analysis on RCC metastases revealed a $35 \%$ rate of LNI and a significant survival difference of 19 vs. 102 months $(\mathrm{P}<0.001)$ when $\mathrm{LN}$ were involved (32). In a series with 175 patients with pulmonary metastasectomy for RCC metastases, LNI was a significant prognostic factor in univariate and multivariate analysis. The hazard ratio for mediastinal LNI was $3.6(\mathrm{P}=0.004)$. The authors used LNI as one of five possible risk factors to score patients into intermediate prognostic group (27). In another small series $(\mathrm{n}=28)$, surgical resection of single LM from different primaries showed superior survival in the group with lobectomy compared to sublobar resection and also less locoregional recurrences when combined with LAD (33).

In a statement on behalf of the ESTS regarding LN involvement during pulmonary metastasectomy, the working group stated that there is no doubt that prognosis is impaired by additional LNI and the best practice would be to exclude, as far as possible, these patients from lung surgery (5).

\section{Risk factors for lymph node involvement}

There is still lacking consensus when to perform a lymphadenectomy during a pulmonary metastasectomy. 

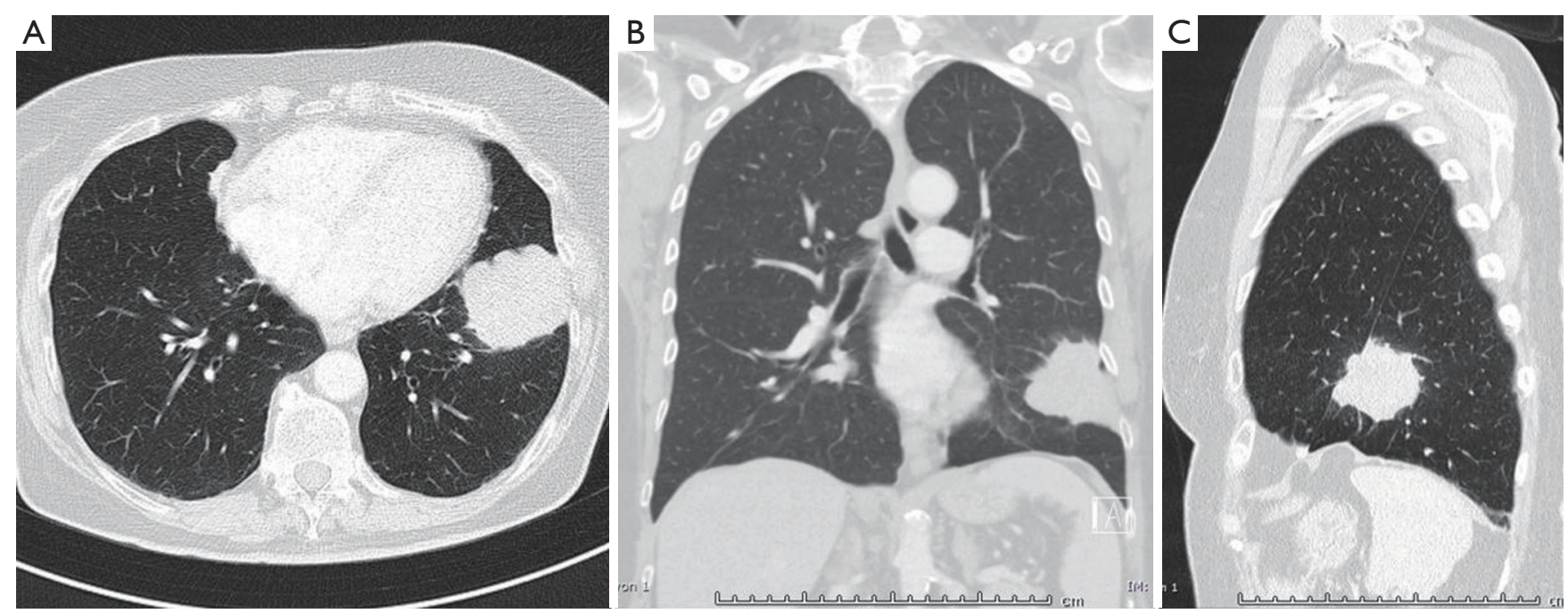

Figure 2 CT scan of an isolated CRC lung metastases in the left lower lobe with interlobar attachment to the upper lobe. Lower lobe resection with LAD revealed a $5.2 \mathrm{~cm}$ adenocarcinoma of colorectal origin with L1 and V1 and intrapulmonary and hilar LNI. CRC, colorectal cancer; LAD, lymphadenectomy; LNI, lymph node involvement.

Central position of the NSCLC lesion is one of the wellknown factors with increased risk for LNI, and has also been described for lung metastases (33). In these patients, a lymphadenectomy might be worth the consideration. There was a significant difference between LNI rates of peripheral versus central CRC pulmonary metastatic locations (18.7\% and $62.5 \%$, respectively; $\mathrm{P}=0.032$ ) (Figures 1 and 2) (34). Furthermore, a higher LNI rate was described for multiple CRC metastases in contrast to single metastasis $(59 \%$ and $44 \%$, respectively; $\mathrm{P}=0.01$ ) (22). However, one must take into consideration the fact that the authors did not exclude N2 disease for lung metastasectomy and this possible selection bias could play a pivotal role in the results. Rectal primary tumor (vs. colon primary tumor), multiple pulmonary lesions and extended lung resection to achieve a tumor-free margins were associated with LNI in a study from 2014 (20). Extended lung resections were performed in centrally located and large metastases (20).

\section{Influence on detection rate}

The number of examined LNs proportionally increase the detection rate of LN metastases in NSCLC (1). However, a comparable number of $\mathrm{LN}$ evaluations does not exist in cases of lung metastasectomy. There is evidence that radical LN dissection increases the number of patients with unexpected $\mathrm{LN}$ involvement (22). The prognosis of $\mathrm{cN} 0$ patients with $\mathrm{LAD}$ and no $\mathrm{LN}$ involvement was significantly better than the prognosis of those without LAD in a study with 429 cases (29). These underline a pseudo stage migration effect from thorough LN examination. The same correlation was described by others for CRC LM and uncertain LN examination compared with $\mathrm{LN}$ dissection (22).

\section{Prognostic influence of lymph node dissection}

As the negative prognostic impact of LNI has been reported in many studies, the logical consequence is that LAD reduces mediastinal recurrence and increase survival rate. Winter et al. (32) evaluated RCC patients with and without radical $\mathrm{LN}$ dissection with matched pairs analysis and found a trend for improved survival in the group undergoing LN dissection ( $\mathrm{P}=0.068)$ (32). In a study of 160 CRC LM cases undergoing radical $\mathrm{LN}$ dissection $(\mathrm{n}=60)$ and no $\mathrm{LN}$ dissection $(\mathrm{n}=100)$, there was no and $23 \% \mathrm{LN}$ recurrence at follow-up (35). One may deduce, that LN recurrence can be prevented by radical removal of ipsilateral LNs.

Another study by Hamaji et al. (36) describes LNI as a prognostic marker. The authors evaluated CRC patients after pulmonary metastasectomy and divided them in 3 groups: patients that underwent systematic nodal dissection (LND) with LNI, LND but no LNI, and no LND. The 5 -year survival was $20.7 \%, 49.3 \%$ and $48.3 \%$, respectively and the corresponding median survivals were $34,58.5$ and 52 months, respectively. LNI significantly correlated with poor prognosis in both univariate and multivariate analyses 
$(\mathrm{P}=0.047$ and $\mathrm{P}=0.0027$, respectively). $\mathrm{LN}$ recurrence occurred at follow-up in all groups. In the LND group with LNI, 8 of 40 patients (20\%) experienced LN recurrence after a median of 42 months, while in LND without LNI, 33 of $279(11.8 \%)$ patients developed LN recurrence after a median of 106 months and in the no LND group 18 of 199 (9\%) patients with $\mathrm{LN}$ recurrence were found. All together LNI was a risk factor for $\mathrm{LN}$ recurrence at follow-up (HR 2.46) (36). As the authors defined LND as harvesting LNs from well-defined locations instead of enbloc dissection of stations this paper is difficult to interpret. Especially the frequency of $\mathrm{LN}$ recurrence at former dissected positions $3 / 8$ is unusual. Unfortunately, they did not compare outcome between the LND and the non-LND groups, but stated that LND is necessary for prognostic purposes.

Contrasting to the aforementioned study results, Londero et al. (37) reports of no additional advantage in terms of survival and recurrence from lymphadenectomy after pulmonary metastasectomy. He analyzed 181 patients after pulmonary metastasectomy with $47.5 \%$ undergoing LAE whereas the others did not have any lymph nodes explored. They did not find any significant difference in the 5-year OS $(\mathrm{P}=0.87)$ or the 5-year cumulative incidence of recurrence $(\mathrm{P}=0.07)$ between the two groups (37). Unfortunately, these results are inconclusive as the patients have been grouped into larger tumors requiring anatomical resection with LAE and small peripheral lesions requiring wedge resection without LAE. Further the authors included a wide range of methods for $\mathrm{LN}$ removal from nodal sampling to lobe specific dissection of $2 \mathrm{LN}$ stations. Therefore, it is not difficult to assume that selection bias and inadequate lymphadenectomy prevented the authors from finding significant differences between the groups. Shiono et al. (38) extracted 683 lobectomy cases from the Metastatic Lung Tumor Study Group of Japan database. While the 5 -year survival rate with increasing levels of LNI decreased: no LNI (53.8\%), up to hilar LNI (39.4\%) and up to mediastinal LNs (30.8\%), respectively, they found no survival difference between various extents of LN removal during lobectomy. As this study included more than 10 different tumor types, the results remain difficult to interpret. The authors concluded that $\mathrm{LN}$ dissection or sampling is necessary to provide prognostic information tor determining postoperative treatment (38).

\section{Recommendations}

According to the ESTS Textbook, some authors state that patients with thoracic nodal disease can be excluded from pulmonary metastasectomy with intent to cure (39). The routine intra-operative assessment of thoracic nodes during metastasectomy should be encouraged, in case of negative preoperative node evaluation. According to the German Guidelines for RCC, resectable lung metastases should be resected with systematic $\mathrm{LN}$ dissection, due to frequent $\mathrm{LN}$ metastasis (Recommendation Grade B, Level of Evidence 3). No clear recommendations concerning LAD during pulmonary metastasectomy exist neither in other German Guidelines, nor in current ESMO-, NCCN- or ESTSGuidelines.

\section{Recommendations to future study projects}

To identify the real benefit of lymphadenectomy during pulmonary metastasectomy, it seems necessary to exclude common confounders. A prospective, randomized study concerning pulmonary metastasectomy has been impossible in the past and will not be applicable for more detailed questions like lymphadenectomy in the future. Therefore, a multi-centric or a nationwide data acquisition of metastasectomy patients in a registry is needed. Based on one histologic entity with a single lesion the analysis of OS, disease specific survival and rate of recurrence (local, mediastinal, distant) can be stratified with or without systematic LAE. The mode of resection (anatomical $v s$. non-anatomical) the use of laser and LNI are some other important variables that need to be considered.

\section{Summary}

LNI is a prognostic factor and is associated with decreased survival after pulmonary metastasectomy in many retrospective studies. The detection rate of additional unexpected LN metastases during pulmonary metastasectomy increases with the radicality of intrathoracic $\mathrm{LN}$ removal, allows more precise prognosis and tailored additional antitumor therapy. Few authors accepted patients with known N2 disease for pulmonary metastasectomy but positive survival rates after additional radical LAE were reported $(24,25)$. Clearance of mediastinal LNs is a prerequisite to prevent undetected LN metastases. Due to the lacking evidence, it is not obligatory in medicolegal terms to perform LAD with metastasectomy. This should be conducted in further studies and larger-scale projects to provide future evidence. 


\section{Acknowledgments}

Funding: None.

\section{Footnote}

Provenance and Peer Review: This article was commissioned by the Guest Editor (Khosro Hekmat) for the series "Pulmonary Metastases" published in fournal of Thoracic Disease. The article has undergone external peer review.

Conflicts of Interest: All authors have completed the ICMJE uniform disclosure form (available at http://dx.doi. org/10.21037/jtd.2020.04.09). The series "Pulmonary Metastases" was commissioned by the editorial office without any funding or sponsorship. SW serves as an unpaid editorial board member of Fournal of Thoracic Disease from May 2019 to Apr 2021. The authors have no other conflicts of interest to declare.

Ethical Statement: The authors are accountable for all aspects of the work in ensuring that questions related to the accuracy or integrity of any part of the work are appropriately investigated and resolved.

Open Access Statement: This is an Open Access article distributed in accordance with the Creative Commons Attribution-NonCommercial-NoDerivs 4.0 International License (CC BY-NC-ND 4.0), which permits the noncommercial replication and distribution of the article with the strict proviso that no changes or edits are made and the original work is properly cited (including links to both the formal publication through the relevant DOI and the license). See: https://creativecommons.org/licenses/by-nc-nd/4.0/.

\section{References}

1. Liang W, He J, Shen Y, et al. Impact of Examined Lymph Node Count on Precise Staging and Long-Term Survival of Resected Non-Small-Cell Lung Cancer: A Population Study of the US SEER Database and a Chinese MultiInstitutional Registry. J Clin Oncol 2017;35:1162-70.

2. Wang W, Chen D, Xi K, et al. Impact of Different Types of Lymphadenectomy Combined With Different Extents of Tumor Resection on Survival Outcomes of Stage I Nonsmall-cell Lung Cancer: A Large-Cohort Real-World Study. Front Oncol 2019;9:642.

3. Internullo E, Cassivi SD, Van Raemdonck D, et al.
Pulmonary metastasectomy: a survey of current practice amongst members of the European Society of Thoracic Surgeons. J Thorac Oncol 2008;3:1257-66.

4. Mokhles S, Macbeth F, Treasure T, et al. Systematic lymphadenectomy versus sampling of ipsilateral mediastinal lymph-nodes during lobectomy for nonsmall-cell lung cancer: a systematic review of randomized trials and a meta-analysis. Eur J Cardiothorac Surg 2017;51:1149-56.

5. García-Yuste M, Cassivi S, Paleru C. Thoracic Lymphatic Involvement in Patients Having Pulmonary Metastasectomy. Incidence and the Effect on Prognosis. J Thorac Oncol 2010;5:S166-9.

6. Welter S, Arfanis E, Christoph D, et al. Growth patterns of pulmonary metastases: should we adjust resection techniques to primary histology and size? Eur J Cardiothorac Surg 2017;52:39-46.

7. Lauweryns JM. The juxta-alveolar lymphatics in the human adult lung. Histologic studies in 15 cases of drowning. Am Rev Respir Dis 1970;102:877-85.

8. Lauweryns JM, Baert JH. Alveolar clearance and the role of the pulmonary lymphatics. Am Rev Respir Dis 1977;115:625-83.

9. Drake RE, Weiss D, Gabel JC. Active lymphatic pumping and sheep lung lymph flow. J Appl Physiol 1991;71:99-103.

10. Riquet M. Thoracic Anatomy I - Chest Wall, Airway, Lungs / The bronchial lymphatics Thorac Surg Clin 2007;17:624-38.

11. Le Pimpec Barthes F, Riquet M, Hartl D, et al. Cervical venous anastomoses of pulmonary lymphatic vessels. Surg Radiol Anat 1997;19:53-5.

12. Riquet M, Le Pimpec Barthes F, Souilamas R, et al. Thoracic duct tributaries from intrathoracic organs. Ann Thorac Surg 2002;73:892-8.

13. Meyer KK. Direct lymphatic connections from the lower lobes of the lung to the abdomen. J Thorac Surg 1958;35:726-33.

14. Riquet M, Hidden G, Debesse B. Abdominal nodal connections of the lymphatics of the lung. Surg Radiol Anat 1988;10:251-2.

15. Wright FW. Enlarged hilar and mediastinal nodes (and especially lower right hilar node enlargement) as a sign of metastasis of a renal tumour. Clin Radiol 1977;28:431-6.

16. Whitson BA, Groth SS, Andrade RS, et al. Extension of survival by resection of asynchronous renal cell carcinoma metastases to mediastinal lymph nodes. J Thorac Cardiovasc Surg 2008;135:1022-8.

17. Kanzaki R, Higashiyama M, Okami J, et al. Surgical 
treatment for patients with solitary metastasis in the mediastinal lymph node from renal cell carcinoma. Interact Cardiovasc Thorac Surg 2009;8:485-7.

18. Pastorino U, Buyse M, Friedel G, et al; International Registry of Lung Metastases. Long-term results of lung metastasectomy: prognostic analyses based on 5206 cases. J Thorac Cardiovasc Surg 1997;113:37-49.

19. Pfannschmidt J, Klode J, Muley T, et al. Nodal involvement at the time of pulmonary metastasectomy: experiences in 245 patients. Ann Thorac Surg 2006;81:448-54.

20. Bölükbas S, Sponholz S, Kudelin N, et al. Risk factors for lymph node metastases and prognosticators of survival in patients undergoing pulmonary metastasectomy for colorectal cancer. Ann Thorac Surg 2014;97:1926-32.

21. Eckardt J, Licht PB. Endobronchial ultrasound-guided transbronchial needle aspiration is a sensitive method to evaluate patients who should not undergo pulmonary metastasectomy. Interact Cardiovasc Thorac Surg 2015;20:482-5.

22. Call S, Rami-Porta R, Embún R, et al. Impact of inappropriate lymphadenectomy on lung metastasectomy for patients with metastatic colorectal cancer. Surg Today. 2016;46:471-8.

23. Seebacher G, Decker S, Fischer JR, et al. Unexpected lymph node disease in resections for pulmonary metastases. Ann Thorac Surg 2015;99:231-6.

24. Renaud S, Alifano M, Falcoz PE, et al. Does nodal status influence survival? Results of a 19-year systematic lymphadenectomy experience during lung metastasectomy of colorectal cancer. Interact Cardiovasc Thorac Surg 2014;18:482-7.

25. Kudelin N, Bölükbas S, Eberlein M, et al. Metastasectomy with standardized lymph node dissection for metastatic renal cell carcinoma: an 11-year single-center experience. Ann Thorac Surg 2013;96:265-70.

26. Welter S, Jacobs J, Krbek T, et al. Prognostic impact of lymph node involvement in pulmonary metastases from colorectal cancer. Eur J Cardiothorac Surg 2007;31:167-72.

27. Meimarakis G, Angele M, Staehler M, et al. Evaluation of a new prognostic score (Munich score) to predict longterm survival after resection of pulmonary renal cell carcinoma metastases. Am J Surg 2011;202:158-67.

28. Renaud S, Falcoz PE, Olland A, et al. Should mediastinal lymphadenectomy be performed during lung metastasectomy of renal cell carcinoma? Interact Cardiovasc Thorac Surg 2013;16:525-8.

29. Nanji S, Karim S, Tang E, et al. Pulmonary
Metastasectomy for Colorectal Cancer: Predictors of Survival in Routine Surgical Practice. Ann Thorac Surg 2018;105:1605-12.

30. Winter H, Meimarakis G, Hoffmann G, et al. Does surgical resection of pulmonary metastases of head and neck cancer improve survival? Ann Surg Oncol. 2008;15:2915-26.

31. Cho JH, Kim S, Namgung M, et al. The prognostic importance of the number of metastases in pulmonary metastasectomy of colorectal cancer. World J Surg Oncol 2015;13:222.

32. Winter H, Meimarakis G, Angele MK, et al. Tumor infiltrated hilar and mediastinal lymph nodes are an independent prognostic factor for decreased survival after pulmonary metastasectomy in patients with renal cell carcinoma. J Urol 2010;184:1888-94.

33. Kamiyoshihara M, Hirai T, Kawashima O, et al. The surgical treatment of metastatic tumors in the lung: is lobectomy with mediastinal lymph node dissection suitable treatment? Oncol Rep 1998;5:453-7.

34. Szöke T, Kortner A, Neu R, et al. Is the mediastinal lymphadenectomy during pulmonary metastasectomy of colorectal cancer necessary? Interact Cardiovasc Thorac Surg 2010;10:694-8.

35. Ali K, Cho S, Jang HJ, et al. Predictive Factors of Thoracic Lymph Node Metastasis Accompanying Pulmonary Metastasis from Colorectal Cancer. Thorac Cardiovasc Surg 2019;67:683-7.

36. Hamaji M, Cassivi SD, Shen KR, et al. Is Lymph Node Dissection Required in Pulmonary Metastasectomy for Colorectal Adenocarcinoma? Ann Thorac Surg 2012;94:1796-800.

37. Londero F, Morelli A, Parise O, et al. Lymphadenectomy during pulmonary metastasectomy: Impact on survival and recurrence. J Surg Oncol 2019;120:768-78.

38. Shiono S, Matsutani N, Okumura S, et al; Metastatic Lung Tumor Study Group of Japan. The prognostic impact of lymph-node dissection on lobectomy for pulmonary metastasis. Eur J Cardiothorac Surg 2015;48:616-21.

39. Treasure T, Scarci M, Nia SP, et al. Pulmonary Metastases. Kuzdzal J, editor. ESTS Textbook of Thoracic Surgery (Crakow). Medycyna Praktyczna 2014.

Cite this article as: Welter S, Gupta V, Kyritsis I. Lymphadenectomy in pulmonary metastasectomy. J Thorac Dis 2021;13(4):2611-2617. doi: 10.21037/jtd.2020.04.09 